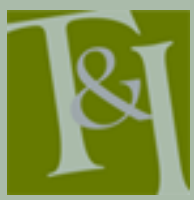

The International Journal for Translation \& Interpreting Research

trans-int.org

\title{
Community interpreting: Asian language interpreters' perspectives
}

\author{
Sophia Ra \\ University of New South Wales \\ s.ra@student.unsw.edu.au
}

Jemina Napier ${ }^{1}$

Heriot-Watt University

j.napier@hw.ac.uk

DOI: ti.105202.2013.a04

\begin{abstract}
With an increasing migrant population worldwide requiring community interpreting services, the role of the community interpreter has been a critical focus in interpreting studies research. As Australia is a multicultural country and one of the leading countries in providing community interpreting services, with a large proportion of immigrants from Asian countries, this paper examines the perspectives of Asian language community interpreters working in Australia on their role and cultural conflicts they can face. Based on an online survey and telephone interviews with Asian language community interpreters accredited by the National

Accreditation Authority for Translators and Interpreters in Australia (NAATI), this paper investigates their perceptions on the interpreter's role and the status of Asian language community interpreting in Australia. The key finding of the study was that Asian language community interpreters predominantly defined their role as a facilitator of communication, and believed that Asian language community interpreting was different from interpreting between two Western or Indo-European languages.
\end{abstract}

Keywords: community interpreting, interpreters, Asian language, Asian language community interpreters, interpreter's role

\section{Introduction}

According to the United Nations' International Migration Report (2006), there are over 200 million migrants around the world. Australia is one of the countries where international migrants constitute a high proportion of the population; up to twenty percent (The United Nations, 2006). As a large proportion of the population come from different countries throughout the world, many different languages other than the national language of English are spoken in Australia. Thus, there has always been a substantial need for interpreters in community settings, such as medical, legal, business and educational contexts for migrants to be fully integrated into the community. Community interpreters can act as a bridge between the Australian mainstream community and the ethnic minority groups or minority language speakers.

This paper focuses on community interpreters who work between

English and Asian languages in Australia. Of the present 200 million migrants around the world, the majority of immigrants come from Asian countries. According to the most recent Australian census (2011), over 12 percent of the Australian population is of Asian descent, predominantly Chinese, Vietnamese, Filipino and Indian. This statistic may be an underestimation when one accounts for non-migrant temporary populations, including international students, short-term working migrants, and illegal 
migrants. This transient population would not appear statistically, but they may need to engage with public services in some way while they are in Australia.

Existing international research in community interpreting has investigated interpreter-mediated communication in various European and Scandinavian language combinations, such as Spanish-English (Angelelli, 2003; Davidson, 2000, 2001), Swedish-Russian (Wadensjö, 1998), DanishEnglish (Jacobsen, 2009), English-German (Pöchhacker, 2007), SpanishArabic (Valero-Garces, 2005), Norwegian-English-Japanese-Chinese (Rudvin, 2007); and between a signed and spoken language (Metzger, 1999; Napier, 2002, 2011; Sanheim, 2003). Little is known, however, about community interpreting when one of the languages is an Asian language. Although it is not possible to define both Asian and Western cultures completely, as both cultures are diverse and varied, Eastern philosophies and religions such as Taoism, Buddhism, Confucianism, Shinto, Hinduism, and Islam have influenced Asian culture (Mindess, 2006). Thus Asian language interpreters may be confronted with certain dilemmas that may conflict with Western values due to this ontology. Therefore, this research on community interpreting involving an Asian language is much needed and vital to our appreciation of the linguistic, cultural and ethical issues involved.

The aim of the study is to build on other similar studies that investigated the perceptions of interpreters (Kelly, 2000; Lee, 2009a), and the research design was partially based on such studies. In order to explore Asian language community interpreters' perceptions of their role, the study was designed in order to investigate the following research questions:

1. What do they think is their role as a community interpreter?

2. Do they consider Asian language community interpreting to be different from any other language combination? And if so, do they believe that distinctive guidelines are needed for Asian language community interpreters?

3. Which features or skills do they think are important to becoming a community interpreter?

\section{Literature Review}

\subsection{Participants' perceptions of the role of the interpreter}

Kelly (2000) and Lee (2009a) both conducted surveys on legal professionals' and interpreters' perceptions of the interpreter's role in the courtroom. In their findings, they found that there might be some cases where court interpreters should interject cultural explanations or linguistic information in the courtroom. However, there were slightly different views between legal professionals and interpreters. According to Kelly (2000), courts expect interpreters to convey cultural information. However, it could be beyond the role of interpreters as not all interpreters are willing to intervene to explain cultural differences when required. Thus Kelly suggests that a differentiated ranking of interpreters with related remuneration could be established.

According to Lee's (2009a) research, based on a survey of Australian court interpreters' views, the Australian-based interpreters tend to be less involved in cultural intervention during their interpreting than those in America when compared with results of Kelly's (2000) survey.

Recently, the role of the interpreter is one of the important issues that is considered by researchers, specifically in relation to how their role in community settings is complex and multifaceted (Angelelli, 2003). Based on 
authentic data of interpreter-mediated interactions in the medical setting, Angelelli argues that interpreters are considered as visible co-participants within interpreter-mediated medical encounters. However, our study reported in this article, focuses on exploring the differences between the Australian mainstream culture and an Asian language speaking culture, and Asian language interpreters' perceptions of their role. Thus, it is important to review the relevant literature on the differences between Western and Asian cultures.

\subsection{Cultural differences between Western and Asian cultures}

For some time it has been recognised that there is a growing number of ethnic minority groups in Australia, and this is still the case. It is inevitable, therefore, that there would be various cultural gaps. In multi-cultural societies, communication is occasionally unsuccessful. Language barriers and cultural differences as expressed through the way people believe are among the main reasons that account for such unsuccessful communication efforts (Wieringen, Harmsen, \& Bruijnzeels, 2002).

In order to examine any possible differences between interpreters working with an Asian language, it is necessary to observe the differences between Western and Asian cultures. Mindess (2006) explains the differences of two cultures largely as collectivism versus individualism. Cultural behaviours in different situations can be explained by this classification. Individuals in collectivist cultures use more indirect styles of communication and silence, which is sometimes to avoid a 'loss of face' in the community (Mindess, 2006, p.179). Also, as relationships and connections among group members are important in collectivist cultures, social hierarchies according to status or age can influence the forms of address, word choices or the manner of speech. On the other hand, in individualistic cultures such as many Western countries, individuals are encouraged to have separate and equal voices and decision making is largely based on what they believe. However, despite all these cultural differences or tendencies, making generalisations is risky. It is improbable that people from the same country or same region have the same views or beliefs. Thus, interpreters should convey content largely based on their understanding of those cultural characteristics and contextual background, or they should seek clarifications for inexplicit information when needed.

\subsection{Cross-linguistic communications between Western and Asian languages}

As Mindess (1999) and Lee (2009c) both explain, many Asian languages such as Chinese or Korean, are 'contextual' languages or 'topic-comment' languages, which have clearly identifiable grammatical structures and orders of sentences. As opposed to English, in which the subject of a sentence generally comes first and the verb comes directly after it, most Chinese or Korean sentences describe a contextual background first (Mindess, 1999). American linguistic typologists $\mathrm{Li}$ and Thompson distinguish topicprominent languages, such as Chinese, Japanese and Korean, from subjectprominent languages, like English. In their view, topic-prominent language structure is maybe independent of the assumption of the basic sentence structure of subject, object and verb. They also point out some features of topic-prominent languages: an absence of the 'dummy subject' like 'it' or 'there', an ellipsis of a subject, and an absence of articles (Li \& Thompson, 1976). As there are situations where the subject or the object of a sentence are omitted in those Asian languages, it would be challenging for an interpreter to find the right subject or an equivalent English word for a particular expression without being informed of a contextual background. 


\subsection{Research on Asian language interpreting in different contexts}

Inter-lingual communication issues have been investigated in criminal courtroom proceedings in Australia, especially on the inexplicit renditions that occurred among Korean speaking witnesses (Lee, 2009b). Based on the analysis of approximately eighty hours of audio recordings of criminal proceedings, Lee explored grammatical and lexical challenges experienced by court interpreters. The main feature is the tendency of the frequent use of ellipsis in Korean, that is to say, subjects, objects or any other major elements of the sentences are often omitted. Furthermore, singularity or plurality is not expressed in Korean when it is not necessary. Thus, in cases where interpreters have no contextual or situational background knowledge, renditions of some sentences into English might be impossible for court interpreters. Lee's data revealed that in some cases, interpreters omitted inexplicit expressions, or they made presumptions about the unexpressed elements and decided what they were, based on their contextual knowledge. Essentially this means that they did not ask for clarification for absent inexplicit expressions if they considered these issues as trivial, perhaps because the information was obvious to them with prior knowledge about the context to which the witness was referring. Lee states that such characteristics of ellipsis is similar in other Asian languages, such as Chinese and Japanese, thus such inter-lingual communication challenges might occur in other Asian language court interpreting in a similar way (Lee, 2009b). Korean witnesses' preference of indirect reported speech was also found (Lee, 2010). Although she focuses on linguistic issues rather than cultural issues, as her research is an empirical discourse analytical study based on the analysis of audio recordings in legal settings, it is a valuable study and made a significant contribution to our understanding of community interpreting between an Asian language and English in a Western institutional context.

Business dialogue interpreting is the site of a research study conducted by Takimoto (2006) based on interviews with Japanese language interpreters. Takimoto examined the functions of Japanese language professional interpreters and their perceptions about intercultural communication. In his study, he argues that the role of business dialogue interpreters should be more flexible as they tend to play a more expanded role, for example they have to intervene during the conversation so that the Japanese client has an opportunity to ask questions or make comments (p.54). Due to the characteristics of business interpreting, interpreters cannot be neutral in some circumstances and have to play multiple roles in business negotiations due to intercultural issues. Based on his interview data, Takimoto (2006) reported that Japanese interpreters stated they occasionally had to expand their role to control turn-taking or stop one of the interlocutors (which was an English speaker in many cases) because 'Japanese are less inclined to cut in due to the cultural difference' (p.54). An additional situation was reported by another of his interpreter participants, who explained that she would add explanations or ask for clarifications because 'Japanese clients do not ask, even when they do not understand something' (p.54). Takimoto comments that the reason that Japanese interpreters are faced with these situations is because 'they are exposed to at least two different sets of potentially different expectations or expectancy norms' (p.54). However, all of these considerations focus solely on business settings, and more general investigations into these questions and issues in other settings are needed.

With regards to the perceptions of the role of the interpreter, contrasted views of different participants on the role of the interpreter, cultural differences between Asian and Western cultures, and previous research on 
Asian language interpreting were examined. To date, as few studies have been conducted on these issues with Asian language interpreting, an overview of the existing literature was provided in order to contrast with the results of this study, and for the findings to be considered in the context of filling a gap in the current literature.

\section{Research Methods}

This research is a mixed-method qualitative study based on an online survey and follow-up telephone interviews with community interpreters in a number of Asian languages: Chinese (Cantonese, Mandarin, and Taiwanese), Filipino, Indonesian, Japanese, Khmer, Korean, Lao, Thai, Burmese and Vietnamese. Those languages were selected by the researchers since they are the most spoken Asian languages at home in Australia (Australian Bureau of Statistics, 2007).

The survey design incorporated open-ended and multiple-choice questions to elicit subjective responses and was divided into three different sections: (1) basic information about interpreter, (2) interpreting qualification and nature of work, and (3) interpreters' own general perceptions about Asian language community interpreters and their roles. Interpreters' personal profiles (such as years of interpreting experience, level of accreditation, whether or not they have had training or study in an interpreting related field, or working settings, etc.) were used for comparing their views or comments on the role of the interpreter, the difficult linguistic or cultural aspects, or the overall perceptions on Asian language community interpreters. The questionnaire had a total of 20 questions (see Appendix 1 for a copy of the survey instrument), which were devised based on the review of the literature.

Interview prompt questions were developed to elicit further information through individual telephone interviews. The questions focused on issues such as experiences on cultural differences between Asian and Western cultures, the need for interpreter's intervention during interpreter-mediated communication, and the efficiency of interpreting related education or training. (See Appendix 2 for a full list of prompt questions)

Participants were recruited through network and snowball sampling (Sadler, Lee, Lim, \& Fullerton, 2010), whereby interpreters were contacted directly through personal researcher networks, and interpreting agencies were also contacted and asked to pass on information to Asian language community interpreters. Information about the online survey was sent to Asian language community interpreters who are listed on the NAATI (National Accreditation Authority for Translators and Interpreters Ltd.) or AUSIT (Australian Institute of Interpreters and Translators Inc.) websites via email, and also distributed to other non-NAATI accredited interpreters who are working as community interpreters through interpreting agencies. As this flyer was only sent via email, interpreters who did not indicate their email addresses on the websites were excluded from this study. The online survey was open for five weeks. The total number of the Asian language interpreters who were contacted directly through the NAATI or AUSIT directories was 452. The survey was distributed via six interpreting agencies. The total number of interpreters who were contacted by those interpreting agencies is difficult to determine.

After collecting the survey responses, respondents who indicated willingness to participate in the second stage of the study were contacted for a follow-up telephone interview. Although there were sixteen respondents who expressed willingness to participate in a telephone interview, only five 
of them sent back the consent form either via email or via post. It was difficult to interpret this low rate of return of the consent form, but it could be only surmised that they might have irregular or unexpected working hours, so they thought it would be difficult to make time to sign, scan and send the consent form back to the researchers and to make designated time available for a telephone interview. Each telephone interview took between twenty and fifty minutes and field notes were taken by one of the researchers. As telephone interviews were not audio-recorded, data from telephone interviews were only employed for supporting the online survey data and not for quoting. The survey and interview data were then qualitatively and thematically analysed to identify key patterns for interpreters' own perceptions about Asian language community interpreting and their roles.

\section{Results and Discussion}

Based on calculations of the reach of the survey via direct contact and interpreting agencies, it is estimated that approximately 500 Asian language community interpreters in Australia received the invitation to participate in the online survey. Forty-five responses were received, giving a response rate of less than $10 \%$, which is a very poor rate (Johnson \& Owens, 2003). Although a flyer of the survey was sent to interpreters who do not have a NAATI accreditation through interpreting agencies, all of the respondents had NAATI accreditation. Thus, it can only be assumed that either community interpreting is a small part of the professional life for nonaccredited interpreters of these Asian languages, or they might consider their inexperience contrary to the goal and intent of the survey. Nonetheless, given that this is the first survey of its kind to focus only on Asian language interpreters in Australia, the results are worth consideration, and may contribute to a better understanding of the working practices of this group. It should be noted, however, that the results are not necessarily generalisable to the wider population of Asian language community interpreters in Australia.

\subsection{Basic information about interpreters}

Forty-five interpreters participated in the online survey. Twenty-five of the respondents were female, sixteen were male, and four of them did not give their gender. The age of the participants were widely scattered throughout all age groups: Of forty-five respondents, twenty were 40-59 years of age, nine were in the 'over sixty' age group, eight were in the 30-39 age group, and four participants were under thirty years of age. Nearly half of the participants (48.8\%) were between forty and fifty-nine years of age and $70.8 \%$ were over forty years old.

The language spread of participants is as seen in

1 , and the majority of participants were born in countries where these languages are used as national languages. However, there were five cases in which the participants were born either in Australia or in England and learned their Asian language as a second language. This group provided some valuable comments on their perceptions on Asian language interpreting from the perspective of someone who does not share the same cultural or migratory background with their non-English speaking clients. 


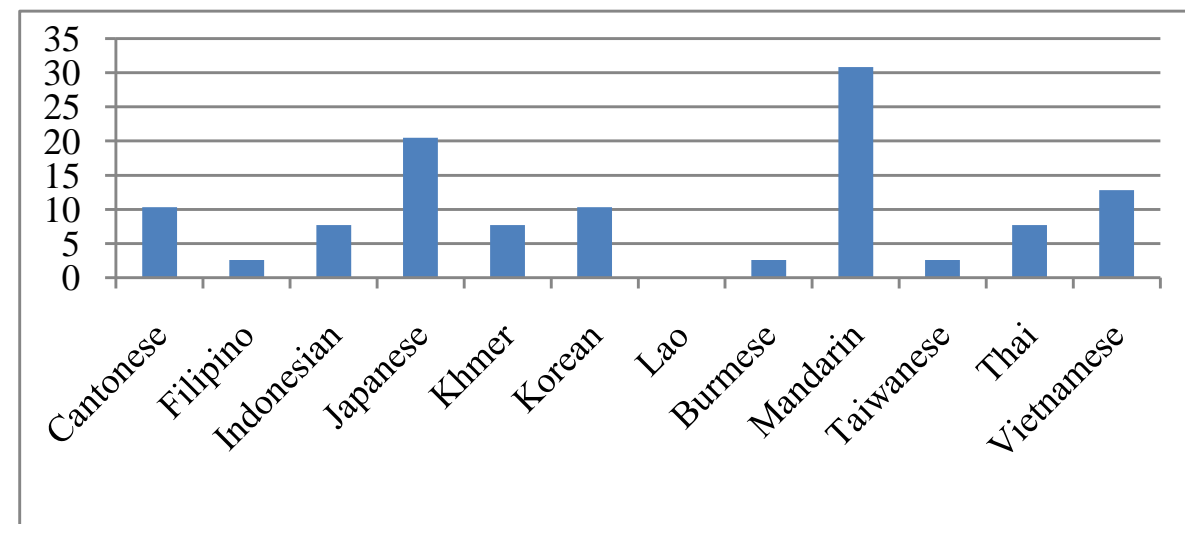

Figure 1. Respondents' languages

The largest group of respondents (35\%) have more than ten years of experience as a community interpreter in Australia. However, relatively novice interpreters, who have less than six years of experience, were also well represented in this survey (19.6\%).

When asked where they work as a community interpreter, most of the respondents selected more than one setting and half of the respondents $(50 \%)$ answered that they work in more than five different settings. One survey respondent explained that he/she still could not receive enough interpreting jobs than needed, even though he/she works in so many different settings. The majority of the participants work in medical/health, legal settings and government bodies (Figure 2). There were only four respondents who answered that they work in one particular setting.

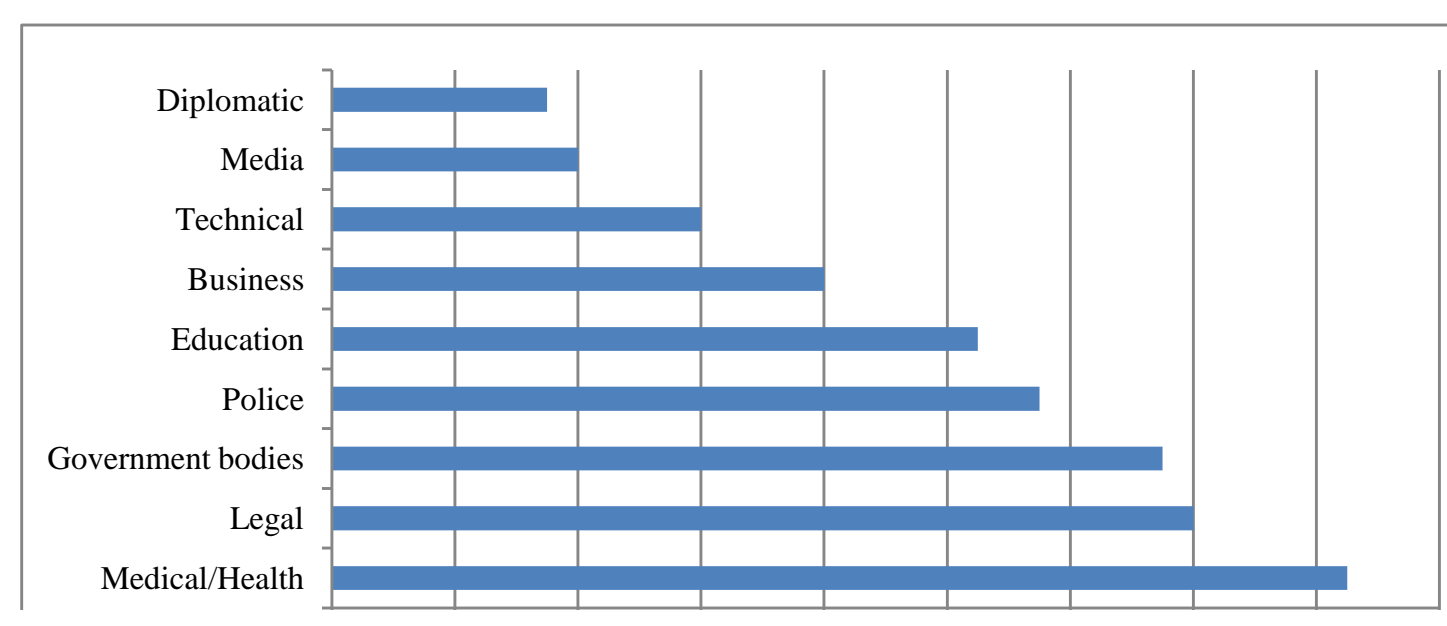

Figure 2. Working settings

\subsection{Accreditation and educational background}

Even if the invitation to this survey was distributed to non-NAATI accredited interpreters through interpreting agencies, all of the respondents were NAATI-accredited interpreters: Of those, $52.5 \%$ were professional interpreters and $47.5 \%$ were paraprofessional interpreters. Five respondents have other overseas accreditations including military interpreter and translator, and diploma of medicine. More than half (57.5\%) have completed or are currently engaged in interpreting-related education or training either in Australia or in other countries. Nevertheless, $92.5 \%$ of the respondents agreed that training or study is necessary for community interpreters. Among thirty-seven respondents who considered that training or study is needed, 
many of them also commented that professional development, which means maintaining or updating their interpreting skills, is as essential as a preservice training, to improve the quality of their interpreting practice and satisfy their customer's needs. This result is consistent with the views noted by previous researchers (Bontempo \& Napier, 2007; Ozolins, 2004).

\subsection{Important features or skills for community interpreters}

In order to elicit the general perception of Asian language community interpreters on important features or skills that might be relevant to their work, eight examples of features were listed in the questionnaire and respondents were asked to indicate which features or skills they felt were most important in community interpreting. Respondents could indicate more than one feature/skill. The results are shown in Table 1.

\begin{tabular}{|l|l|}
\hline Linguistic skills and comprehension (English) & $\mathbf{8 7 . 5 \%}$ \\
\hline $\begin{array}{l}\text { Linguistic skills and comprehension (LOTE, Languages } \\
\text { Other Than English) }\end{array}$ & $85 \%$ \\
\hline Ability to manage interaction smoothly & $72.5 \%$ \\
\hline Communication skills & $70 \%$ \\
\hline Knowledge of primary participants' cultures & $62.5 \%$ \\
\hline Taking ethical responsibility & $60 \%$ \\
\hline Technical interpreting skills & $57.5 \%$ \\
\hline Shared background (cultural or migratory) with clients & $32.5 \%$ \\
\hline
\end{tabular}

Table 1. Important features or skills for community interpreters

Since respondents were able to choose as many features as they feel apply, seven out of eight features were selected by more than half of the respondents. This can be explained by the fact that most of the features listed were considered to be important factors for community interpreters to facilitate communication. More than $70 \%$ of participants believe that linguistic skills and general communication skills are the most critical features. It is interesting to note, however, that only $32.5 \%$ consider that having a shared cultural or migratory background with clients is vital for community interpreters. It is particularly notable that the small group of respondents who have an Asian language as their second language did not choose the last trait of shared background. One respondent from this particular group commented that knowledge and understanding of primary participants' cultures is sufficient.

\subsection{Perceptions of the interpreter's role and Asian language community interpreting}

Respondents were asked to select the best description of the role of the interpreter in community settings among these representations: a translation machine, a facilitator of communication, a cultural expert, a language expert, an advocate for a client, and a cultural mediator or broker. Although it was a multiple choice question and they were able to choose more than one answer, most of the respondents $(91.9 \%)$ regard themselves as a facilitator of communication and nearly half of them (45\%) select only one answer as a facilitator of communication. Only four respondents consider themselves as a translation machine and three as an advocate for a client. Unfortunately, as those who consider their role as a translation machine or an advocate did not provide supplementary comments to this question, the reason why they 
designated those answers could not be surmised. Interestingly though, there was no connection between those results and the educational background or years of experiences as a community interpreter. On the contrary, some respondents added comments on the online survey that they consider the role as a translation machine or an advocate is not their role:

The interpreter uses the knowledge and understanding of two languages to help facilitate the communication between two parties. I don't think of myself as an advocate for a client but rather to assist both parties to communicate and understand the other. I also don't see myself as a translation machine, which may not hear or see the nuance in the speech. (Respondent 34)

Interpreters should be qualified and competent to interpret. That is our expertise. Culture and language are not our expertise, and require additional training and qualification. Also, by definition interpreters are impartial. Advocacy and brokerage require impartiality to be set aside. (Respondent 7)

As seen on Respondent 7's comment above, the opinion that community interpreters would not be necessarily cultural mediators or cultural brokers is predominant. It is interesting to note that this view of Asian language community interpreters on their role is rather inconsistent with the views noted by previous researchers (Angelelli, 2003; Kelly, 2000; Singy \& Guex, 2005), in which interpreters are considered as a more active and visible participant. However, there were quite contrary views from other respondents from online survey. $24.3 \%$ of respondents consider that their role is a cultural mediator/broker and $27 \%$ regard themselves as cultural experts. Some respondents believe that community interpreters cannot perform their main role, which is a facilitator of communication here again, without bridging two cultures:

My role is to facilitate communication between two parties by a faithful rendering of the message, which requires a good understanding of both languages and cultures (Respondent 45)

This result shows that Asian language community interpreters consider basically that their main role is to facilitate communication between two parties who speak two different languages. However, with regards to consideration of themselves as cultural mediators or cultural brokers, they have contrasting views. As shown in Lee's (2009a) research based on a survey of Australian court interpreters' views, Australian-based interpreters tend to be less involved in cultural intervention during their interpreting than those in America when compared with the results of Kelly's (Kelly, 2000) survey. Thus, the reluctance of Asian language community interpreters to consider their role as a cultural mediator is in line with the results of Lee's study. This may be because they are not comfortable with the terms 'cultural mediators' or 'cultural brokers' rather than the role itself. As we had not offered definitions of those terms and about half of the respondents did not have any educational background in interpreting studies, the terms might have been unfamiliar and/or unclear to respondents.

Also, in her study, Angelelli (Angelelli, 2004a, 2004b) discovered a contradiction between what interpreters said and what they did in reality. That is to say, interpreters often said that they were impartial, but the author observed that they were not. Torikai (2010) also indicates that interpreters sometimes play a role of bridging cultural barriers in intercultural communication without being conscious of their role as 'cultural clarifiers' 
(p. 87). Likewise, Asian language community interpreters who responded to this survey may practice cultural mediation in their work, but simply do not wish to admit it on this questionnaire perhaps due to a fear of being judged or uncertainty as to whether their behaviour is professional and ethical. Further empirical investigations of Asian language interpreter-mediated communication are needed, so that the results of this survey can be compared with what happens in practice.

In order to determine what Asian language interpreters believe to be cultural aspects requiring intervention and explanation by an interpreter, participants were asked to select which cultural aspects may lead to difficult interpreting situations. Cultural aspects that were listed in this question were drawn from the literature (Kelly, 2000; Lee, 2009a). The response rates are shown in Table 2.

\begin{tabular}{|l|r|}
\hline $\begin{array}{l}\text { Differences of cultural customs and behaviour patterns } \\
\text { (including social/cultural rituals) }\end{array}$ & $67.6 \%$ \\
\hline $\begin{array}{l}\text { Culture-related terms and expressions (including dialects and } \\
\text { colloquial language) }\end{array}$ & $59.5 \%$ \\
\hline $\begin{array}{l}\text { Cultural concepts (such as individualism vs. collectivism and } \\
\text { Confucianism) }\end{array}$ & $37.8 \%$ \\
\hline Meaning of gestures & $21.6 \%$ \\
\hline
\end{tabular}

Table 2. Cultural aspects that make interpreting situations difficult in community settings

As shown in this table, respondents consider both verbal and non-verbal features as cultural aspects, which might lead to difficult situations in interpreter-mediated communication. One respondent also commented, during the telephone interview, that as most Asian languages are contextual languages, you cannot get the message, in many cases, without understanding non-verbal expressions.

With regards to their perceptions on Asian language community interpreting in Australia, the largest number of respondents (43.2\%) state that they are not sure whether Asian language interpreting might be different from interpreting between other Western language combinations. $37.8 \%$ of respondents believe that Asian language interpreting is different and $18.9 \%$ declare that there is no difference. Many of them who agree that Asian language interpreting is different point to the syntax of languages, the beliefs of community members, and the ways of speaking as different aspects.

However, when they were asked whether it is necessary to have special guidelines for Asian language interpreters, almost half of the respondents $(48.6 \%)$ agreed that they are not essential. $29.7 \%$ of the respondents agreed on the necessity of distinctive guidelines for Asian language interpreters and $21.6 \%$ stated that they do not know. Although more respondents consider that Asian language interpreting might be different from interpreting between any other Western languages, not many of them felt that special guidelines for Asian language interpreters are required. One survey respondent's comment gives some insight into why they believe that Asian language interpreting is not different:

It is not only different from other interpreting between two Western or Indo-European languages, but also different among Asian language interpreting. For example, Vietnamese culture is different from Japanese, Cambodian, Thais, etc. (Respondent 8) 
Community interpreters already have a code of ethics to abide by and Asian language interpreters are able to address situations, in which they can face linguistic or cultural conflicts, based on their common sense or experiences and through professional development. No code of ethics can predict every conceivable scenario. Thus they believe that distinctive guidelines for Asian language interpreters will not be able to resolve each and every issue even if guidelines were established. A number of codes indeed advise that professional judgement is required to apply the general guideline of the code (Hale, 2007).

\section{Conclusion}

Having reviewed the results above, it can be seen that from the small sample who responded to our survey, that Asian language community interpreters consider themselves as facilitators of communication. As they have experience as interpreters and recognise linguistic and cultural differences between Asian and Western cultures, nearly $40 \%$ of the respondents believe that Asian language community interpreting is different from interpreting between other Western language combinations; but they do not consider that distinct guidelines for Asian language interpreters are needed.

The survey results also demonstrate that Asian language community interpreters assume that linguistic and comprehension skills in both English and LOTE, communication skills and cultural knowledge or understanding are the most important features for community interpreters. However, shared migratory or cultural background with clients was not regarded as a critical feature for Asian language interpreters, which contradicts the findings of Angelelli's (2003) study, in which interpreters were considered as cultural brokers or cultural bridges. However, as Angelelli's study was conducted with Spanish-English interpreters in America, and her study participants were non-professional interpreters who did not necessarily have any educational background in interpreting, the different cultural values must be taken into account.

These findings have potential implications for the training and education of interpreters. Demands for interpreting between English and an Asian language are increasing in Australia, but interpreters themselves still have conflicted views on the interpreters' role as a cultural mediator. Therefore interpreter education or professional development programs could develop practical guidelines for community interpreters on how they can overcome cultural challenges during their interpreting without being unprofessional or unethical. As Ozolins (2004) indicates in his survey report, many practitioners expressed a great interest in professional development opportunities. Thus, a professional development program for practitioners who are interested in developing and improving their interpreting skills and cultural background knowledge, could be provided more systematically. Regular conferences or seminars, or the setting up of a network among Asian language community interpreters could assist them to share their knowledge and strategies and to improve the quality of interpreting.

As this study is based on an online survey and a telephone interview, results are limited in terms of generalisation. Therefore, further studies need to be conducted based on authentic data, such as interpreter-mediated interviews in government organisations, medical consultations, or proceedings in legal settings, to explore how Asian language interpreters manage cultural differences within different contexts, and how they actually 
manage their role as compared to how they report their perceptions of their role.

Although the data collected in this study provided a small sample of respondents, the results give us some valuable initial insight into Asian language community interpreters' perceptions of their role. Although there are studies on community interpreters who work with other languages, this study was intended as a gathering of perceptions of Asian language community interpreters only, and appears to be the first of its kind in Australia. The findings are not ground-breaking, but there are some interesting points of note, which are worth taking into consideration.

Thus, the findings of this study raise issues that need to be investigated further in order to advance the professional status of Asian language community interpreters in Australia and worldwide. A comparative study on Asian language community interpreting with previous studies, which were conducted with other Western spoken languages or signed languages, informed by empirical studies, may contribute to improving the quality of intercultural communication by Asian language community interpreters in Australia. It would also be valuable to investigate how cultural aspects or difficulties affect the quality of interpreting and how Asian language interpreters overcome culturally ethical challenges. 


\section{Reference List}

Angelelli, C. (2003). The visible co-participant:the interpreter's role in doctor-patient encounters. In M. Metzger, S. Collins, V. Dively \& R. Shaw (Eds.), From topic boundaries to omission: New research on interpretation (pp. 3-26 ). Washington DC: Gallaudet University Press.

Angelelli, C. (2004a). Medical interpreting and cross-cultural communication New York : Cambridge University Press

Angelelli, C. (2004b). Revisiting the interpreter's role: A study of conference, court, and medical interpreters in Canada, Mexico, and the United States. Amsterdam ; Philadelphia John Benjamins Pub. .

Australian Bureau of Statistics. (2007). Ancestry by Country of Birth of Parents - Time Series Statistics (2001, 2006 Census Years) - Australia. Australian Bureau of Statistics.

Australian Bureau of Statistics. (2011). Ancestry by Country of Birth of Parents. Australian Bureau of Statistics.

Bontempo, K., \& Napier, J. (2007). Mind the gap! A skills analysis of sign language interpreters. The Sign language translator and interpreter, $1(2), 275-299$.

Davidson, B. (2000). The interpreter as institutional gatekeeper: The sociallinguistic role of interpreters in Spanish-English medical discourse. Journal of Sociolinguistics, 4(3), 379-405.

Davidson, B. (2001). Questions in cross-linguistic medical encounters: The role of the hospital interpreter. Anthropological Quarterly, 74(4), 170178.

Hale, S. (2007). Community Interpreting. Basingstoke: Palgrave Macmillan. Jacobsen, B. (2009). The community interpreter: A question of role. Hermes - Journal of Language and Communication Studies, 42, 155-166.

Johnson, T., \& Owens, L. (2003). Survey response rate reporting in the professional literature. Paper presented at the Annual meeting of the American Association for Public Opinion Research, Nashville. http://www.allacademic.com/meta/p116171_index.html

Kelly, A. M. (2000). Cultural parameters for interpreters in the courtroom. In R. P. Roberts, S. E. Carr, S. E. Carr \& A. Dufour (Eds.), The critical link 2: Interpreters in the community: Selected papers from the Second International Conference on Interpreting in Legal, Health, and Social Service Settings, 19-23 May 1998 (pp. 131-148). Vancouver, BC, Canada,: Amsterdam ; Philadelphia : John Benjamins Pub Co

Lee, J. (2009a). Conflicting views on court interpreting examined through surveys of legal professionals and court interpreters. Interpreting: international journal of research and practice in interpreting, 11(1), 3556.

Lee, J. (2009b). Interpreting inexplicit language during courtroom examination. Applied Linguistics, 30(1), 93-114.

Lee, J. (2009c). When linguistic and cultural differences are not disclosed in court interpreting. Multilingua, 28(4), 379-402.

Lee, J. (2010). Interpreting reported speech in witnesses' evidence. Interpreting: international journal of research and practice in interpreting, 12(1), 60-82.

Li, C. N., \& Thompson, S. A. (1976). Subject and Topic: A New Typology of Language. In C. N. Li (Ed.), Subject and Topic (pp. 457-489).

London/New York: Academic Press.

Metzger, M. (1999). Footing shifts in an interpreted mock interview. In E. Winston (Ed.), Storytelling and Conversation: Discourse in Deaf Communities. Washington, D.C.: Gallaudet University Press. 
Mindess, A. (1999). Reading between the signs: A practical approach to cultural adjustments. Paper presented at the RID 1999 National Convention Proceedings, Silver Spring, MD.

Mindess, A. (2006). Reading between the signs: Intercultural communication for sign language interpreters (Second ed.). London: Nicholas Brealey Publishing.

Napier, J. (2002). Sign language interpreting: linguistic coping strategies.: England: Douglas McLean.

Napier, J. (2011). 'It's not what they say but the way they say it.' A contect analysis of interpreter and consumer perceptions of signed language interpreting in Australia. International Journal of the Sociology of Language, 2011(207), 59-87.

Ozolins, U. (2004). Survey of Interpreting Practitioners: Report. VITS Language Link good corporate citizenship program. Melbourne: VITS Language Link.

Pöchhacker, F. (2007). Coping with culture in media interpreting. Perspectives: Studies in Translatology, 15(2), 123-142.

Rudvin, M. (2007). Professionalism and ethics in community interpreting: The impact of individualist versus collective group identity. Interpreting: International journal of research and practice in interpreting, 9(1), 47-69.

Sadler, G., Lee, H., Lim, R., \& Fullerton, J. (2010). Recruitment of hard-toreach population subgroups via adaptations of the snowball sampling strategy. Nursing \& health sciences, 12(3), 369-374.

Sanheim, L. R. (2003). Turn exchange in an interpreted medical encounter. In M. Metzger, S. Collins, V. Dively \& R. Shaw (Eds.), From topic boundaries to omission: New research on interpretation (pp. 27-54). Washington, DC: Gallaudet University Press.

Singy, P., \& Guex, P. (2005). The interpreter's role with immigrant patients: Contrasted points of view. Communication \& medicine, 2(1), 45-51.

Takimoto, M. (2006). Interpreters' role perceptions in business dialogue interpreting situations. Monash university linguistics papers, 5(1), 47-57.

The United Nations. (2006). The International Migration Report 2006: A Global Assessment.

Torikai, K. (2010). Conference interpreters and their perception of culture: From the narratives of Japanese pioneers. Translation and Interpreting Studies, 5(1), 75-93.

Valero-Garces, C. (2005). Doctor-Patient Consultations in Dyadic and Triadic Exchanges. Interpreting: international journal of research and practice in interpreting, 7(2), 193-210.

Wadensjö, C. (1998). Interpreting as interaction. London/New York : Longman

Wieringen, J., Harmsen, J., \& Bruijnzeels, M. A. (2002). Intercultural communication in general practice. The European journal of public health, 12(1), 63-68.

\section{Notes}

1. Jemina Napier is also an Adjunct Professor at Macquarie University. The research was conducted by Sophia Ra under the supervision of Jemina Napier at Macquarie University. 


\section{Appendix 1. Survey Questionnaire}

\section{Personal information}

1. Gender

2. Male/Female

3. Age group

4. Language that you interpret from and into in community-based interpreting

5. Country of birth?

6. How many years have you lived in Australia?

\section{Interpreting qualification and nature of work}

7. NAATI accreditation level

8. Do you have any other overseas accreditation?

9. Have you completed or are you currently engaged in an interpreting related education?

10. Do you think training or study is important for community-based interpreters?

11. Which features or skills, in your view, are the most important for community-based interpreters? (Please tick as many as apply) Linguistic skills and comprehension (English)

- $\quad$ Linguistic skills and comprehension (LOTE, Language Other Than English)

Technical interpreting skills

Ability to manage interaction smoothly

Taking ethical responsibility

Knowledge of primary participants' cultures

Communication skills

- $\quad$ Shared background (cultural or migratory) with clients

- $\quad$ Other (Please specify)

12. How many years have you worked as a community-based interpreter in Australia?

13. Have you worked as a community-based interpreter in any other countries?

14. Give details about settings where you generally work as a community-based interpreter (Please tick as many as apply)

Business

Diplomatic

Education

Government bodies

Legal

Media

Medical/Health

Police

Technical

Other (Please specify)

\section{Perceptions on Asian language community interpreters}

15. Which describes best, in your view, the overall role of the interpreter in community settings? And why? (Please tick as many as apply) As a translation machine As a facilitator of communication 
As a cultural expert

As a language expert

As an advocate for the client

As a cultural mediator/broker

16. In your view, what cultural aspects make interpreting situations

difficult in community settings? (Please tick as many as apply.)

Culture-related terms and expressions (including dialects and

colloquial languages)

Differences of cultural customs and behaviour patterns (including social/cultural rituals)

Meaning of gestures

- Cultural concepts (such as individualism vs. collectivism and

Confucianism )

Other (Please specify)

17. Who would you ask for help or advice from when you have had a difficult situation while you were interpreting? (Please tick as many as apply)

18. Do you generally talk to your customers (both non-English speaking client and English speaking professionals) before your assignment starts? And why? (Please comment)

19. What do you think is the benefit in talking to your client (either nonEnglish speaking clients or English speaking professionals) before the appointment? (Please tick as many as apply)

- $\quad$ Comfortable atmosphere during interpreting

- $\quad$ To establish trust with the client

- $\quad$ Opportunity to observe the clients' interpersonal nature

- $\quad$ Opportunity to establish clients' expectations with regard to the interpreter's role

Other (Please specify)

20. Do you think Asian language interpreting is different from other interpreting between two western or Indo-European languages?

21. Do you think we need special guidelines for Asian language interpreters who work in community settings in Australia? 


\section{Appendix 2. Telephone interview questions}

1. Do you often experience a situation where you find the differences of Asian and Western cultures?

2. When you talk to your customers, especially your non-English speaking client, before your assignment starts, if they ask you some advice or your opinion, what would you do?

3. To what extent, do you think, interpreters can intervene during the communication? For example, there has been a misunderstanding from one of the parties and you have noticed it, but others haven't?

4. In what ways, do you think, we can share our knowledge or experiences?

5. Do you think community interpreters should interpret non-verbal expressions as well?

6. Do you think that an explanation of cultural differences during dialogue is the role of the interpreter? 